\title{
4.7-T diffusion tensor imaging of acute traumatic peripheral nerve injury
}

\author{
Richard B. Boyer, PhD, ${ }^{1,2}$ Nathaniel D. Kelm, MS, ${ }^{1,3}$ D. Colton Riley, BS, ${ }^{2}$ Kevin W. Sexton, MD,, 4 \\ Alonda C. Pollins, MLI, ${ }^{2}$ R. Bruce Shack, MD, ${ }^{2}$ Richard D. Dortch, PhD, ${ }^{1,3}$ Lillian B. Nanney, PhD, ${ }^{2}$ \\ Mark D. Does, PhD, ${ }^{1,3}$ and Wesley P. Thayer, MD, PhD ${ }^{1,2}$
}

\begin{abstract}
Departments of ${ }^{1}$ Biomedical Engineering and ${ }^{2}$ Plastic Surgery, Vanderbilt University Medical Center; ${ }^{3}$ Vanderbilt University Institute of Imaging Science, Nashville, Tennessee; and ${ }^{4}$ Department of Surgery, University of Arkansas for Medical Sciences, Little Rock, Arkansas
\end{abstract}

\begin{abstract}
Diagnosis and management of peripheral nerve injury is complicated by the inability to assess microstructural features of injured nerve fibers via clinical examination and electrophysiology. Diffusion tensor imaging (DTI) has been shown to accurately detect nerve injury and regeneration in crush models of peripheral nerve injury, but no prior studies have been conducted on nerve transection, a surgical emergency that can lead to permanent weakness or paralysis. Acute sciatic nerve injuries were performed microsurgically to produce multiple grades of nerve transection in rats that were harvested 1 hour after surgery. High-resolution diffusion tensor images from ex vivo sciatic nerves were obtained using diffusion-weighted spin-echo acquisitions at 4.7 T. Fractional anisotropy was significantly reduced at the injury sites of transected rats compared with sham rats. Additionally, minor eigenvalues and radial diffusivity were profoundly elevated at all injury sites and were negatively correlated to the degree of injury. Diffusion tensor tractography showed discontinuities at all injury sites and significantly reduced continuous tract counts. These findings demonstrate that high-resolution DTI is a promising tool for acute diagnosis and grading of traumatic peripheral nerve injuries.
\end{abstract}

http://thejns.org/doi/abs/10.3171/2015.6.FOCUS1590

KEY WORDS diffusion tensor imaging; diffusion tensor tractography; MRI; neurography; peripheral nerve injury; nerve transection; neurotmesis

$\mathrm{N}$ EUROTMESIS, or peripheral nerve transection, is a common but difficult to distinguish diagnosis following traumatic injury. Electrodiagnostic studies and clinical examination are standard practice for peripheral nerve assessment, but neither can perfectly discriminate a severe nerve laceration from a self-resolving axonotmetic or neurapraxic injury in the acute setting..$^{16}$ Additionally, Wallerian degeneration may take up to 4 weeks before these injuries can be accurately diagnosed in the absence of exploratory surgery. ${ }^{14}$ This is also true for peripheral nerve repair, in which it may take up to a year, depending on the site and severity of injury, before targets are reinnervated and surgical outcome is known. Although a "watch and wait" approach might appear to be the optimal conservative management, the time following a severe Sunderland fourth- or fifth-degree peripheral nerve lesion is critical to recovery. ${ }^{9}$ Failure to microsurgically repair a nerve in a timely manner may result in irreversible muscle atrophy, weakness, paralysis, or formation of painful traumatic neuromas. ${ }^{9}$

Peripheral nerve injury is one of the few acute neurological disorders in which imaging is not the standard of care. Transected nerves can be identified accurately with the aid of high-resolution ultrasound. ${ }^{10,29}$ However, traumatic injuries have proven difficult to diagnose using ultrasonography, because of large hematomas, extensive skin lacerations, edema, and disruption of the normal anatomy. ${ }^{10}$ Furthermore, no studies have shown the ability of high-resolution ultrasonography to reveal early nerve regeneration, which ultimately would indicate whether addi-

ABBREVIATIONS AUC = area under the curve; DTI = diffusion tensor imaging; DTPA = diethylene triamine pentaacetic acid; FA = fractional anisotropy; $M D=$ mean diffusivity; MRN = MR neurography; PBS = phosphate-buffered saline; PFA = paraformaldehyde; $\mathrm{ROC}=$ receiver-operator characteristic; $\mathrm{ROI}=$ region of interest; SNR = signalto-noise ratio; $\lambda \|=$ axial diffusivity; $\lambda \perp=$ radial diffusivity. 
tional surgical intervention is required ${ }^{28}$ MR neurography (MRN) leverages the microstructural features of axonal bundles to produce enhancement of peripheral nerves in MRI. ${ }^{6,12,15,17,26}$ Nerves normally exhibit a moderate signal on T2-weighted sequences, but they become hyperintense following injury, because of prolongation of the T2 relaxation time. ${ }^{1}$ Although this can be a sensitive marker of peripheral nerve pathology, conventional T2-weighted MRI lacks specificity for type and severity of nerve injury. 5,7 In the most severe cases of nerve injury involving gaps, MRN is capable of detecting nerve discontinuity. ${ }^{11}$ However, more than half of all high-grade nerve transections have minimal or no gap present.

Diffusion tensor imaging (DTI) is an MRI sequence that leverages the anisotropic diffusion of water molecules through axons to aid visualization of neural tracts and assessment of microstructural features. DTI parameters, such as fractional anisotropy (FA), mean diffusivity (MD), and individual eigenvalues $(\lambda 1-\lambda 3)$ are able to quantify microstructural characteristics of nerves, such as axon density and myelin thickness. ${ }^{35}$ In the CNS, DTI has been particularly useful in detecting axonal pathology in traumatic brain injury and spinal cord injury. ${ }^{2,8}$ Multiple investigators have evaluated DTI for detecting peripheral nerve crush and traction nerve injuries, which show significant changes in FA following injury. $22,23,25,31$ However, limited data exist on high-grade nerve lacerations and there are no prior animal studies of DTI following microsurgical coaptation.

The ability to noninvasively assess axonal integrity could have vast implications in the management of peripheral nerve disorders. In the case of traumatic nerve injury, an accurate noninvasive measurement of axonal continuity could assist in surgical planning and postoperative management. ${ }^{15}$ Iatrogenic peripheral nerve injury may also occur during certain medical procedures, such as nerve blocks, leading to an expensive and time-consuming neurological and musculoskeletal workup. ${ }^{19}$ DTI may be able to quickly diagnose these conditions and expedite therapy. Last, there has been growing interest in neuromodulatory therapy, which leverages the peripheral nerve tracts as targets for electrophysiological interventions. As peripheral nerve interventions are developed, it will be important to have noninvasive tools for diagnosis and management of adverse events (e.g., needle injuries). The specificity of DTI for axonal tracts makes it an ideal tool for guiding these cases.

Peripheral nerve research may also benefit from DTI. Nerve regeneration models are highly dependent on behavioral testing and histology, which require large animal trials with multiple end points and error-prone assessments, such as the sciatic functional index. ${ }^{3,20}$ High-resolution DTI may not only provide real-time, noninvasive measurements of axon viability but also allow for several intermediate measurements of nerve recovery in survival studies. This could reduce the large number of animals required in nerve regeneration studies, in addition to providing greater understanding of the regeneration time line.

As DTI can be used to accurately detect acute peripheral nerve crush and traction injuries, we hypothesized that high-resolution DTI also could be used to accurately dis- tinguish acute peripheral nerve transection lesions from intact nerve in the absence of macroscopic discontinuity. We tested this hypothesis using a complete and partial rat sciatic nerve injury model and high-resolution DTI at 4.7 $\mathrm{T}$. The partial nerve transection injury model was chosen to determine the correlation of axonal disruption and DTI parameters in the acute injury setting. This model was also chosen to mimic experimental axonal-level nerve repair, such as polyethylene glycol fusion, in which a subset of proximal and distal axon stumps are believed to recover anatomical and functional connection immediately following treatment. ${ }^{18,27}$ Immediately following injury, sciatic nerves were microsurgically repaired to maintain close approximation of the epineurium, mimicking a lesion that would be difficult to detect with conventional MRI. In addition to determining the diagnostic capabilities of DTI in high-grade nerve lesions, this study provides a baseline for postoperative evaluation of peripheral nerve repairs using DTI.

\section{Methods \\ Experimental Design}

The objective of this study was to evaluate the accuracy of DTI parameters, including fractional anisotropy (FA), mean diffusivity (MD), radial diffusivity $(\lambda \perp)$, and individual eigenvalues in detecting acute peripheral nerve transection. This study was performed using a rat sciatic nerve injury model with sham $(n=6)$, partial $(n=9[n=3$ per group]), and complete transection injuries $(n=6)$. DTI was performed on preparations of excised sciatic nerve and amputated hind limbs following fixation.

\section{Animal Model}

Adult female Sprague-Dawley rats aged 10-12 weeks (200-250 g; Charles River Lab) were used in the experiments. Rats were housed in a central animal care facility and provided food and water ad libitum. All study procedures involving animals were approved by our institutional animal care and use committee.

\section{Sciatic Nerve Injury}

In each rat, the left hindquarter was shaved and the lateral thigh was incised from the sciatic notch to the popliteal fossa. The biceps femoris was reflected to expose the sciatic nerve. Surrounding fascia was sharply dissected and the sciatic nerve mobilized. The injury was created by completely or partially transecting the sciatic nerve with extra fine Vannas microsurgical scissors (RS-5640; Roboz Surgical Instrument Co.). In partial transections, approximately $25 \%$ or $50 \%$ of the nerve diameter was advanced onto the cutting edge of the scissor under high magnification. Sham nerves were mobilized and not transected. Microsurgical suturing of the epineurium was performed on injured nerves to control for variable nerve gaps and to stabilize injury sites. The muscle was repaired and skin incision closed in a subgroup of rats for in situ imaging $(\mathrm{n}=8)$. All rats were killed at 1 hour after injury with perfusion fixation using $4 \%$ paraformaldehyde (PFA) in phosphate-buffered saline (PBS). The sciatic nerve was excised, straightened on a wood dowel, and incubated in 
$4 \%$ glutaraldehyde/ $0.5 \%$ PFA in PBS at $4^{\circ} \mathrm{C}$. Hindquarter amputation was performed on the subgroup of rats with closed wounds and the amputated leg was incubated in $4 \%$ glutaraldehyde $/ 0.5 \%$ PFA in PBS at $4^{\circ} \mathrm{C}$.

\section{Tissue Sample Preparation}

After 24 hours of postfixation, excised nerves were placed in PBS plus $2 \mathrm{mM}$ Gd-diethylene triamine pentaacetic acid (DTPA) (Magnevist, Bayer HealthCare) at $4^{\circ} \mathrm{C}$ for at least 24 hours before imaging. For imaging, excised nerves were trimmed to approximately $1 \mathrm{~cm}$ in length (with the injury site centered) and placed in glass capillary tubes (2-mm outer diameter) filled with a perfluropolyether liquid (Fomblin, Solvay Solexis) for susceptibility matching, preventing tissue dehydration, and a signal-free background. For higher throughput, 6 excised sciatic nerves in a hexagonal arrangement were imaged simultaneously. Hindlimb samples were postfixed for 1 week, followed by at least 1 week of washing in PBS plus $2 \mathrm{mM}$ Gd-DTPA before imaging. For imaging, hind limbs were placed in MR-compatible tubes filled with perfluoropolyether liquid.

\section{Diffusion Tensor MRI}

MRI was performed on a 4.7-T, 31-cm horizontal bore, Agilent DirectDrive scanner (Agilent Technologies) using a 38-mm Litz quadrature coil (Doty Scientific) for radiofrequency transmission and reception. For excised nerve imaging, the FOV was $9.6 \times 9.6 \times 12 \mathrm{~mm}^{3}$ and the matrix size was $96 \times 96 \times 32$ for a nominal resolution of $100 \times$ $100 \times 375 \mu^{3}$ (375 $\mu \mathrm{m}$ along the nerve). For hindlimb imaging, the FOV was $48.0 \times 25.6 \times 28.8 \mathrm{~mm}^{3}$ and the matrix size was $192 \times 128 \times 144$, for a nominal resolution of $250 \times 200 \times 200 \mu^{3}(250 \mu \mathrm{m}$ along the nerve $)$. DTI data were acquired using a 3D diffusion-weighted spin-echo sequence with a TR/TE of 170/23.0 msec and 12 signal averages for excised nerves, and a TR/TE of 170/22.1 msec and 2 signal averages for hind limbs. Diffusion weighting was achieved with a $\delta / \Delta$ of $4 / 12 \mathrm{msec}$, a prescribed b value of $2000 \mathrm{sec} / \mathrm{mm}^{2}$, and 6 directions. One $\mathrm{b}=0$ image was acquired, for a total of 7 images in a scan time of approximately 12 hours.

\section{DTI Postprocessing and Analysis}

Image data reconstruction and diffusion tensor calculations were performed using in-house written code in MATLAB (Mathworks). 3D image volumes were zero padded twice in each direction during reconstruction from k-space data. Diffusion tensors were estimated voxelwise using a linear least-squares approach. From the diffusion tensor, DTI metrics, including FA and MD, axial diffusivity $(\lambda l)$, and $\lambda \perp$, were computed voxelwise. DTI tractography was performed using the ExploreDTI toolbox. ${ }^{21}$

\section{Histology}

After completion of imaging, the distal end of the nerve was trimmed to provide a fresh stump and chloromethylbenzamido-DiI membrane-labeling paste (NeuroTrace CM-DiI Tissue Labeling Paste; Life Technologies/Thermo Fisher Scientific), a carbocyanine dye derivative, was applied to the nerve ending, using a 27-gauge needle. The dyed nerve ending was embedded in petroleum jelly to prevent leakage of the tracer and placed in PBS-sodium azide $(0.02 \%)$. The nerve was stored at $37^{\circ} \mathrm{C}$ in the dark for 2 weeks to allow for tracing in the proximodistal direction through the injury site.

Segments of proximal and distal nerve were postfixed in $10 \%$ neutral buffered formalin for 24 hours and paraffin embedded. Formalin-fixed, paraffin-embedded tissues were sectioned at $5 \mu \mathrm{m}$, placed on slides, and warmed overnight at $60^{\circ} \mathrm{C}$. Slides were deparaffinized with xylene and rehydrated with graded alcohols, ending in distilled water. Prolong Gold antifade reagent with DAPI (Life Technologies/Thermo Fisher Scientific) was applied to the slide and a coverslip was placed over the slide, which was allowed to dry and was stored at $4^{\circ} \mathrm{C}$ until ready to view.

Digital images of slides were acquired with a Nikon AZ100 M widefield fluorescence microscope (Nikon Corp.). CM-DiI fluorescence was observed using a Cy-3 fluorescence filter with 548-nm/561-nm excitation/emission wavelengths. All sections were viewed with a $5 \times$ Zeiss Plan fluorescence objective (Carl Zeiss AG) without coverslips.

\section{Statistical Analysis}

Prism 6 (GraphPad Software) was used for plots and statistical calculations. Quantitative region of interest (ROI) statistics were performed with Student t-tests corrected for multiple comparisons using the Holm-Sidak method, with significance determined as at an $\alpha$ of 0.05 .

\section{Results Injury Validation}

Various degrees of high-grade peripheral nerve injury were evaluated in this study using complete and partial transections of rat sciatic nerves followed by ex vivo DTI. The severity of nerve injury was predicted at time of surgery with careful microsurgical nerve dissection and verified with histology. CM-DiI membrane label was observed in all sham and transected nerves and, therefore, could not be used as a direct marker of injury severity, most likely due to tracer leakage or membrane approximation. However, during evaluation of the proximal nerve sections, we observed that injured nerves had greater axon caliber compared with sham nerves. Completely transected nerves were found to have significantly larger mean axon caliber compared with sham nerves $(\mathrm{p}<0.01)$ (Fig. 1C). As observed in diffuse axonal injury in brain white matter, cytotoxic edema occurs rapidly after damage to the axolemmal membrane, causing axonal swelling. ${ }^{24}$ In this study, we observed that mean axon caliber also scaled with the predicted severity of nerve injury (Fig. 1D), with moderate correlation of predicted percentage of injured axons and mean axon caliber $\left(r^{2}=0.59 ; p=0.002\right)$. There was wide variability in axon caliber in nerves predicted to be $75 \%$ transected, which we suspect was due to surgical error. Removing the nerves that were $75 \%$ transected from the statistical analysis improved the correlation of predicted injury severity and mean axon caliber $\left(r^{2}=0.82\right.$; $\mathrm{p}=0.0001)$. 


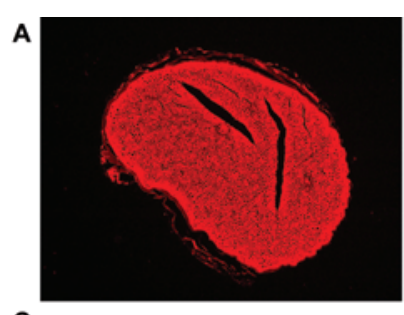

C
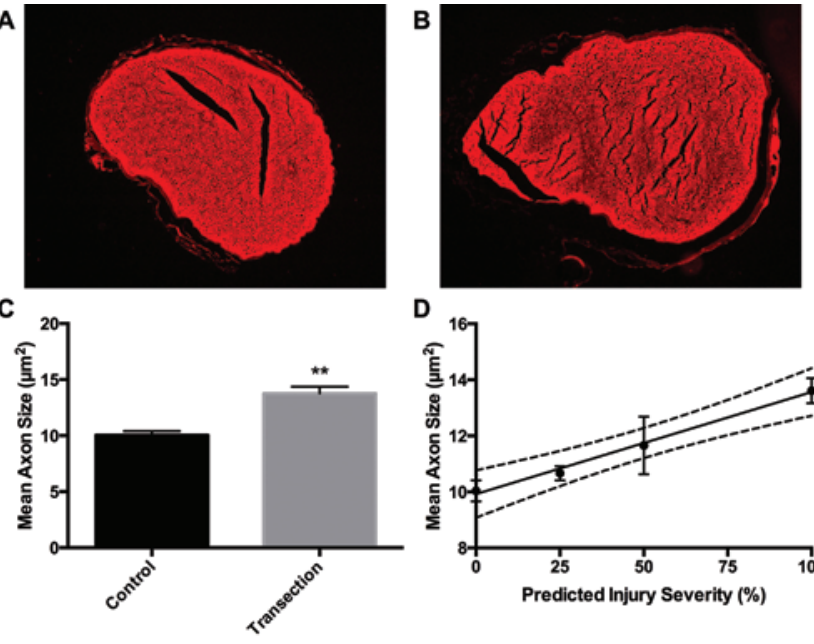

D

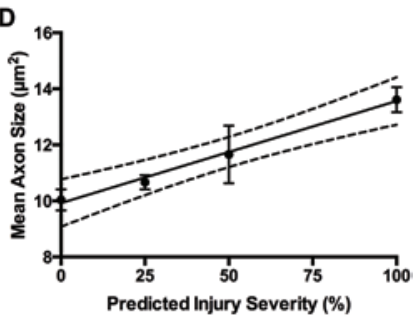

FIG. 1. Predicted nerve injury severity correlated with proximal axon caliber. A and B: Representative fluorescent microscopy images of CM-Dil membrane label in (A) sham and (B) completely transected sciatic nerves. Axoplasm is visible as holes in the center of red membrane rings. C: Axon caliber was measured $3 \mathrm{~mm}$ proximal to the injury site for all axons visible in the axial cross-sections of sham and completely transected nerves (mean $\pm S E M ; n=6$ ). $D$ : Mean axon caliber correlated moderately with the predicted injury severity $\left(r^{2}=0.59 ; n=15\right.$; $p=0.002$ ). However, correlation strongly improved after removing the nerves predicted to be $75 \%$ transected $\left(r^{2}=0.82 ; n=12 ; p=0.0001\right)$. Dashed lines indicate the $95 \% \mathrm{Cl} .{ }^{* *} \mathrm{p}<0.01$, unpaired t-test with Welch correction.

\section{DTI of Completely Transected Nerves}

Diffusion tensor images of excised sciatic nerves were evaluated at 3 ROIs: the injury site and $3 \mathrm{~mm}$ proximal and $3 \mathrm{~mm}$ distal to the injury site. To prevent partial volume effects, a margin of $100 \mu \mathrm{m}$ was excluded from the ROI. This also prevented the inclusion of suture material in ROI calculations, since knots are limited to the outermost epineurium. FA, MD, and eigenvalues were measured for each ROI (Fig. 2A-C). As expected, the FA was significantly decreased at the injury sites of transected compared with sham rats $(\mathrm{p}<0.01)$. Although not as strong as at the injury site, the proximal ROI of transected nerves also had lower FA compared with sham rats $(\mathrm{p}<$ 0.01). Whereas the drop in FA at the injury site indicates axonal loss, the proximal ROI changes were most likely due to edema following nerve injury, causing an increase in extracellular fluid compartment and an increase in axonal edema, which would be expected to decrease FA while simultaneously increasing MD and eigenvalues. Although MD was significantly lower at the injury site $(\mathrm{p}<0.01)$ and unchanged in the proximal and distal ROIs, the minor eigenvalues showed an increasing trend at all ROIs in transected nerves compared with sham rats (Fig. 2C). Additionally, radial diffusivity, calculated as $(\lambda 2+\lambda 3) / 2$, was significantly increased in the injury site $(\mathrm{p}<0.05)$ and proximal ROI $(\mathrm{p}<0.05)$ of transected nerves compared with sham rats. We observed larger variance in distal ROI quantitative measurements, which may be due to variation in fascicular branching at this level in the sciatic nerve. Receiver-operator characteristic (ROC) curves were evaluated for injury-site DTI measurements (Fig. 2D). Injurysite FA produces a near-perfect ROC curve (area under the

curve [AUC] 1.000; p < 0.001), but a larger sample size would be required to prevent Type II error. Table 1 shows the calculated sensitivities and specificities of different FA thresholds for diagnosing complete nerve transection.

Continuous fiber tractography was performed with a maximum $45^{\circ}$ angle and threshold FA of 0.49 , corresponding to $1 \mathrm{SD}$ above the mean FA of all injury sites. Continuous tracts were observed throughout the body of each sham nerve (Fig. 3A), whereas there were multiple discontinuities present in transected nerves (Fig. 3B), particularly at the injury site. Fewer tracts were visible in the proximal segments of injured nerves, most likely due to reduced FA from tissue edema. Additionally, the number of continuous tracts seeded proximally and passing through the injury site and distal ROI were significantly lower in transected nerves compared with sham rats ( $\mathrm{p}<0.01$; Fig. $3 \mathrm{C}$ ). Principal diffusion vectors at injury sites were also observed to be more heterogeneous than sham nerves, with greater variability in diffusion direction (Fig. 4).

\section{DTI of Partially Transected Nerves}

For determining the degree to which partial severance of the sciatic nerve could be distinguished with DTI, we imaged partially transected nerves that were graded at the time of surgery as $25 \%, 50 \%$, or $75 \%$ nerve severance and validated histologically, as described under Methods. DTI parameters were evaluated at the injury ROI for each injured nerve and at the center of each sham nerve. Mean FA was significantly reduced in all injured nerves compared with sham nerves (Fig. 5A). However, there were no significant differences between the levels of injury. Mean FA of sham nerves was strongly correlated with the nerve axon count, but this relationship was not present in injured nerves (Fig. 5B). Interestingly, no significant differences were found in MD (Fig. 5C) or $\lambda \| l$ (Fig. 5E), as has been observed in prior studies of predegenerated contusive nerve injuries. ${ }^{31}$ Following Wallerian degeneration, sciatic nerves have been shown to have normal MD but markedly decreased $\lambda \|$ and increased radial diffusivity. ${ }^{4}$ In this study, minor eigenvalues $(\lambda 2, \lambda 3)$ and radial diffusivity were profoundly increased at all injury sites immediately following injury (Fig. 5E).

Although there was no significant correlation between eigenvalues and predicted degree of injury, there was a weak negative correlation between $\lambda 2$ and $\lambda \perp$ to proximal axon caliber (Fig. 5G). Neither parameter was correlated to nerve axon count in sham or injured nerves.

\section{DTI of Hind Limbs}

As proof of concept, ex vivo imaging was performed on amputated rat hind limbs with sham or complete transection injuries. Tractography was performed with multiple seed points spaced $1 \mathrm{~mm}$ apart along the axial length of the sciatic nerve. We found that seeding from a single axial slice, as was done in all excised nerves, resulted in short tracts, even at low FA thresholds. By seeding from multiple axial slices along the nerve, we were able to capture the length of the nerve while continuing to observe discontinuities at all injury sites (Fig. 6A and B). Quantitative DTI measurements were calculated at multiple ROIs 
A

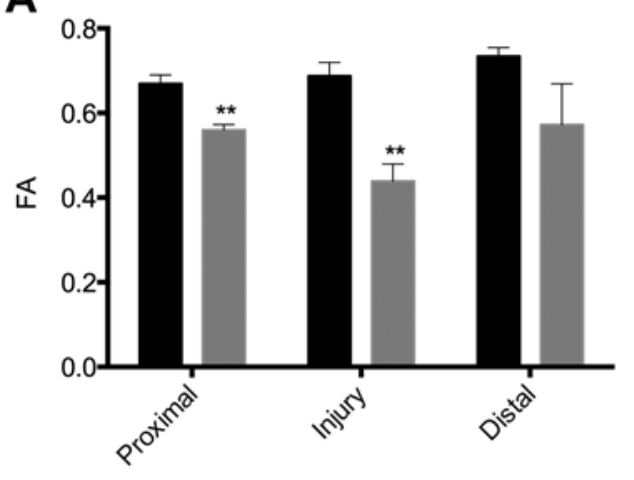

C

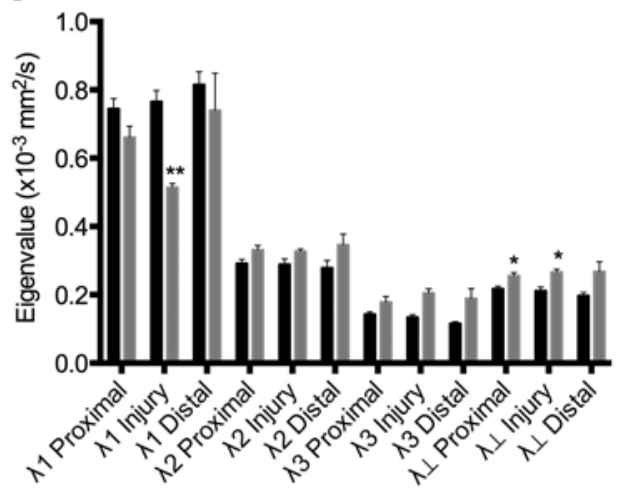

B

Control

Transection

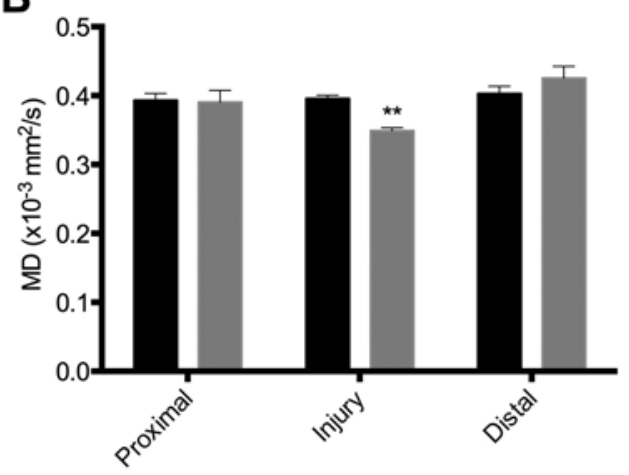

Control

Transection

FIG. 2. FA enabled detection of complete nerve transection with high sensitivity and specificity. A: FA was reduced at and proximal to injury sites (mean $\pm S E M ; n=6$ ). B: MD was reduced only within injury sites (mean $\pm S E M ; n=6$ ). C: Independent eigenvalues $(\lambda 1, \lambda 2, \lambda 3)$ and $\lambda \perp$ were measured at proximal, injury, and distal regions. Axial diffusivity ( $\lambda \|)$ was reduced within injury sites and $\lambda \perp$ was increased at and proximal to injury sites (mean $\pm S E M ; n=6$ ). D: ROC curve analysis of FA shows high sensitivity and specificity for detection of any nerve injury (AUC 1.00; $n=21 ; p<0.001$ ). Unpaired t-tests corrected for multiple comparisons using the Holm-Sidak method. ${ }^{*} p<0.05 ;{ }^{* *} p<0.01$.

(i.e., the injury site and proximal $[1,3$, and $5 \mathrm{~mm}]$ and distal $[1,3$, and $5 \mathrm{~mm}]$ from the injury site). The additional proximal and distal ROIs were included in this analysis to compare effects of surrounding tissue inflammation and edema. Similar to excised nerves, FA was significantly reduced at the injury site of transected nerves $(p<0.01)$ and, to a lesser extent, in the proximal and distal ROIs (Fig. 6C). We hypothesized that external tissue effects should be similar within the entire surgical site, whereas sciatic nerve injury effects should diminish with increasing distance from the injury site. This was confirmed by our measurements of FA at multiple distances from each injury site (Fig. 6D), in which the FA appears to reach an asymptote at $3 \mathrm{~mm}$, but remains significantly lower than in sham animals $(\mathrm{p}<0.05)$.

\section{Discussion}

We present findings that show high-resolution DTI enables detection of acute peripheral-nerve severance injuries in the absence of macroscopic nerve discontinuity. We also provide a baseline measurement of DTI parameters following microsurgical neurorrhaphy, which builds a foundation for the use of DTI in postoperative management. Importantly, intralesional FA was a highly sensitive and specific marker of nerve injury even with partial nerve

TABLE 1. Sensitivity and specificity of FA for all nerve injuries

\begin{tabular}{cccccc}
\hline FA Threshold & Sensitivity, $\%$ & $95 \% \mathrm{Cl}$ & Specificity, $\%$ & $95 \% \mathrm{Cl}$ & Likelihood Ratio \\
\hline$<0.5630$ & 100.0 & $78.20-100.0$ & 100.0 & $54.07-100.0$ & \\
\hline 0.6197 & 100.0 & $78.20-100.0$ & 83.33 & $35.88-99.58$ & 6.000 \\
\hline$<0.6406$ & 100.0 & $78.20-100.0$ & 66.67 & $22.28-95.67$ & 3.000 \\
\hline$<0.6838$ & 100.0 & $78.20-100.0$ & 50.00 & $11.81-88.19$ & 2.000 \\
\hline$<0.7127$ & 100.0 & $78.20-100.0$ & 33.33 & $4.327-77.72$ & 1.500 \\
\hline$<0.7219$ & 100.0 & $78.20-100.0$ & 16.67 & $0.4211-64.12$ & 1.200 \\
\hline
\end{tabular}



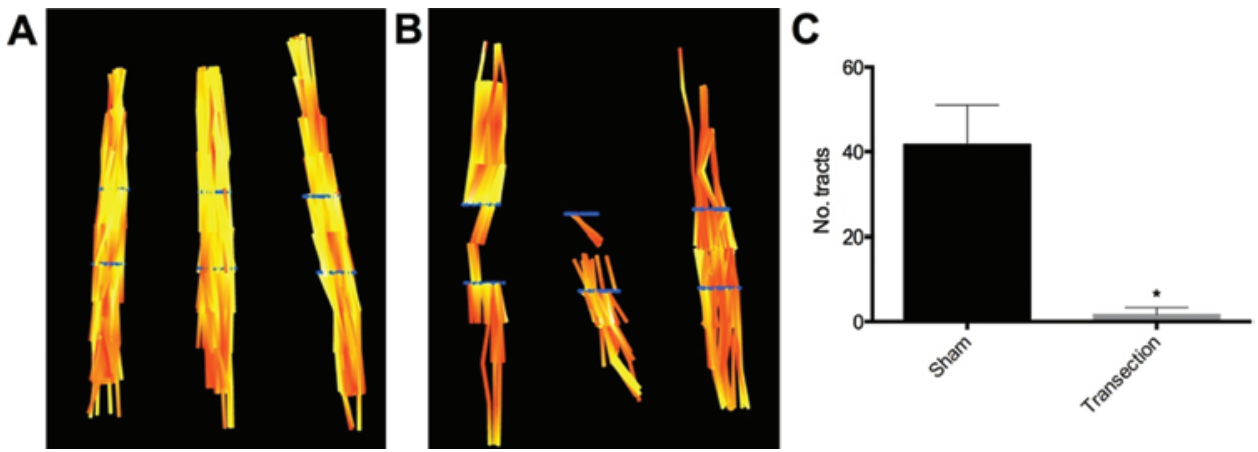

FIG. 3. Tract continuity diminished in completely transected nerves. A and B: Diffusion tensor tractography in sham (A) and completely transected (B) sciatic nerves. Blue lines indicate seed points for tractography located at $3 \mathrm{~mm}$ proximal and distal to the injury sites. C: Continuous tract counts through the injury region were significantly reduced in completely transected nerves (mean \pm SEM; $n=6$ ). Unpaired t-test with Welch correction. ${ }^{\star} p<0.01$.

lacerations, and, unlike nerve crush injuries, there was a concurrent increase in radial diffusivity. This could be the result of immediate membrane and myelin disruption present in severance but not contusive nerve injuries.

Radial diffusivity following injury was also correlated to the degree of nerve severance. If proximal axon caliber is an accurate reflection of axonal swelling following nerve injury, then this could indicate that $\lambda 2$ and $\lambda \perp$ are inversely proportional to the degree of nerve severance following injury. Although this is counterintuitive, since both parameters were significantly increased following transection, it is likely that there are multiple microstructural factors contributing to these changes. One possible explanation is that severance largely increases $\lambda 2$ and $\lambda \perp$ because of membrane and myelin disruption while simultaneously, and to a lesser extent, decreasing because of immediate axonal swelling. These findings are supported by prior studies of DTI following sciatic nerve transection or traction injuries ${ }^{22,23}$ and suggest that high-resolution DTI may be capable of both diagnosis and grading of traumatic peripheral nerve injuries.

Prior studies evaluating DTI of peripheral nerve injuries have focused on the effects of crush or contusion with blunt force trauma. ${ }^{23,25,31}$ Although nerve crush is a significant cause of morbidity, typically occurring as a surgical complication, traumatic nerve injuries often involve partial or complete neurotmesis that can result in lifelong disability if not promptly diagnosed and microsurgically repaired. ${ }^{9}$ Providing a new method of detecting nerve transection could prevent delays in surgical repair that lead to irreversible weakness, loss of function, and other undesirable sequelae. Additionally, DTI could be highly valuable as a tool for assessing nerve repairs and determining when a revision surgery is necessary.

There are multiple limitations in this study. Most importantly, the majority of this study was conducted on excised sciatic nerves that had been cleaned and cross-linked in fixative prior to imaging. Fixation is known to reduce
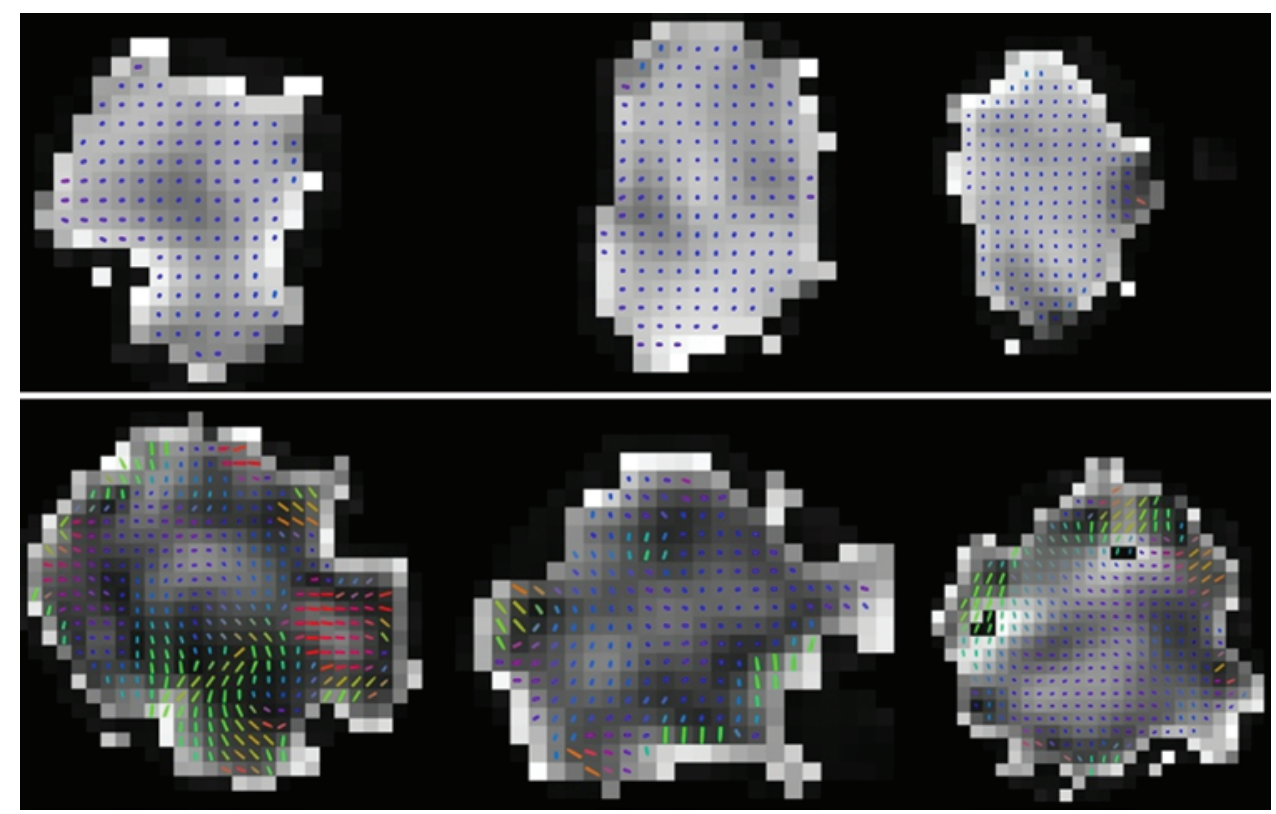

FIG. 4. Principal diffusion vectors are heterogeneous at transection sites. Principal diffusion vector glyphs overlaying the axial FA map of the injury region in sham (upper) and completely transected (lower) nerves. Colors indicate vector direction. Lighter regions of the map indicate higher $F A$. 
A
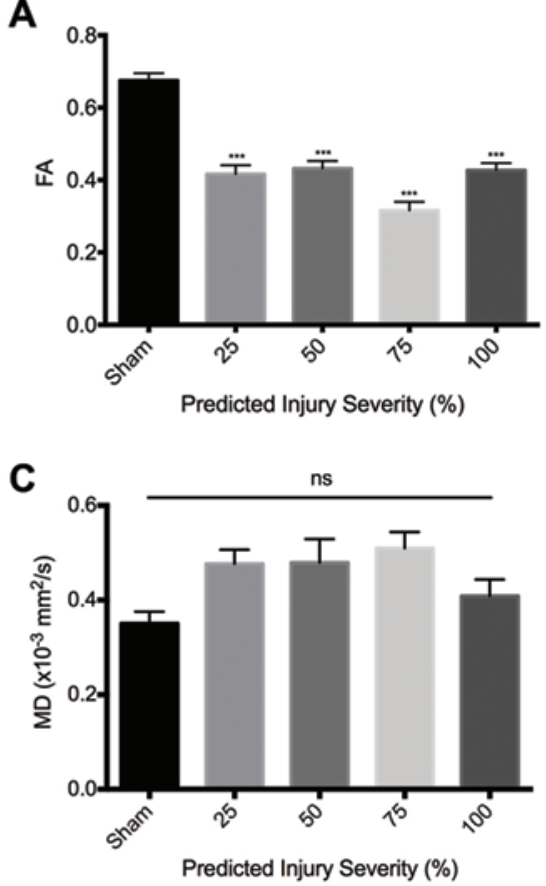

E

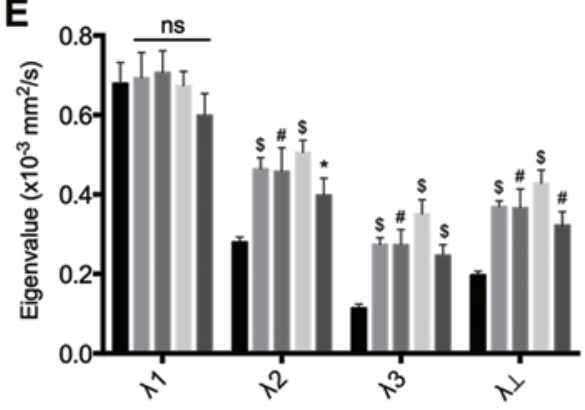

Sham $25 \%-50 \%-75 \%-100 \%$
B
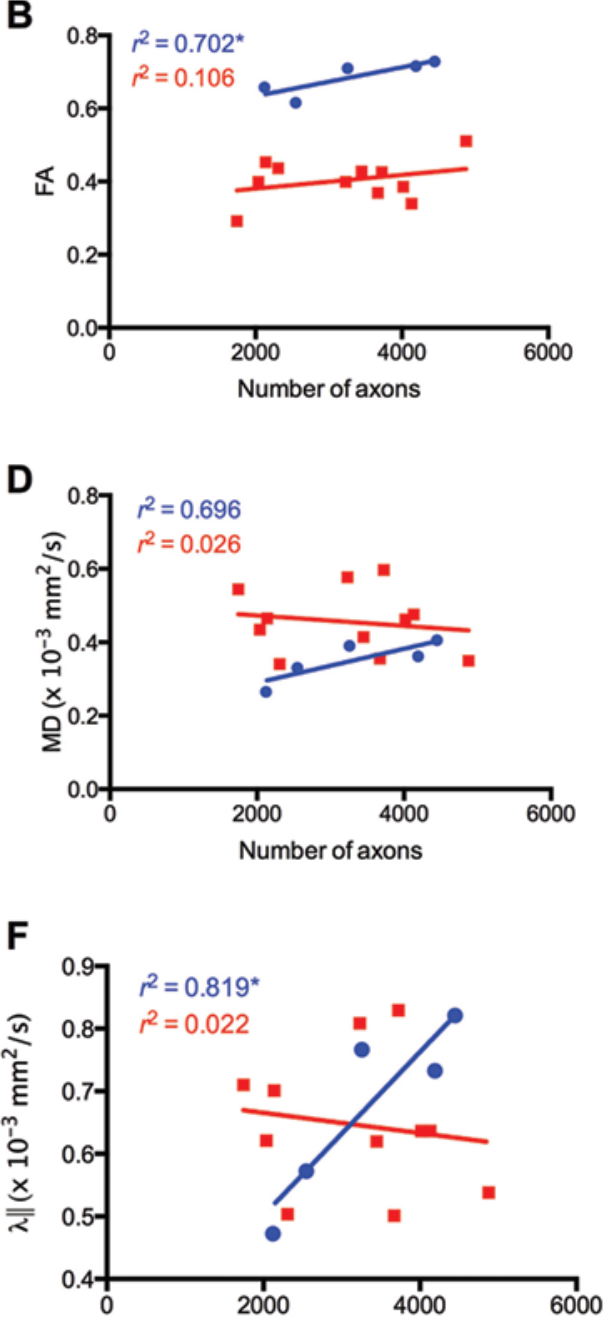

Number of axons

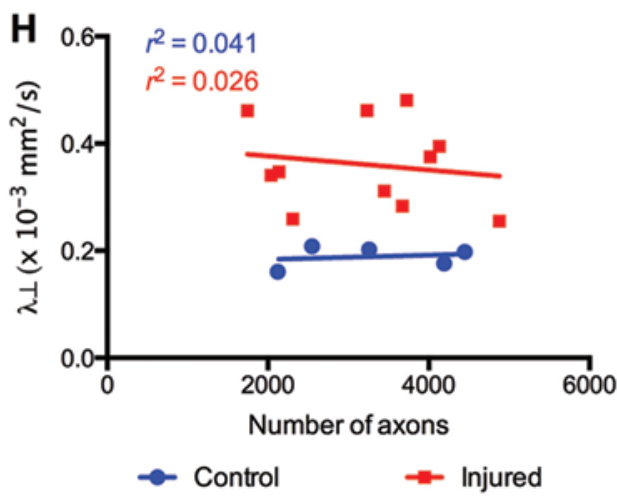

FIG. 5. FA and $\lambda \perp$ detected all partial injuries and $\lambda \perp$ correlated with injury severity. Quantitative analysis of DTI parameters in sham, partial, and complete sciatic nerve transection injuries. A: FA was similarly reduced in the injury region of all complete and partial nerve injuries (mean $\pm S E M ; n=21$ ). $C$ : MD showed a nonsignificant increase in all injury groups. $E$ : Minor eigenvalues and $\lambda \perp$ were significantly increased in the injury region in all injury groups (mean $\pm S E M ; n=21$ ). $B, D, F$, and $H$ : There was no correlation of FA (B), MD (D), $\lambda \|(F)$, or $\lambda \perp(H)$ measured at injury sites with the number of axons in injured nerves. $G: \lambda \perp$ was negatively correlated to axon caliber in injured nerves and indirectly to injury severity $\left(r^{2}=0.357\right)$. One-way ANOVA corrected for multiple comparisons with Tukey test. ${ }^{*} p<0.05 ; \# p<0.01 ; \$ p<0.001 ;{ }^{* *} p<0.0001$. 

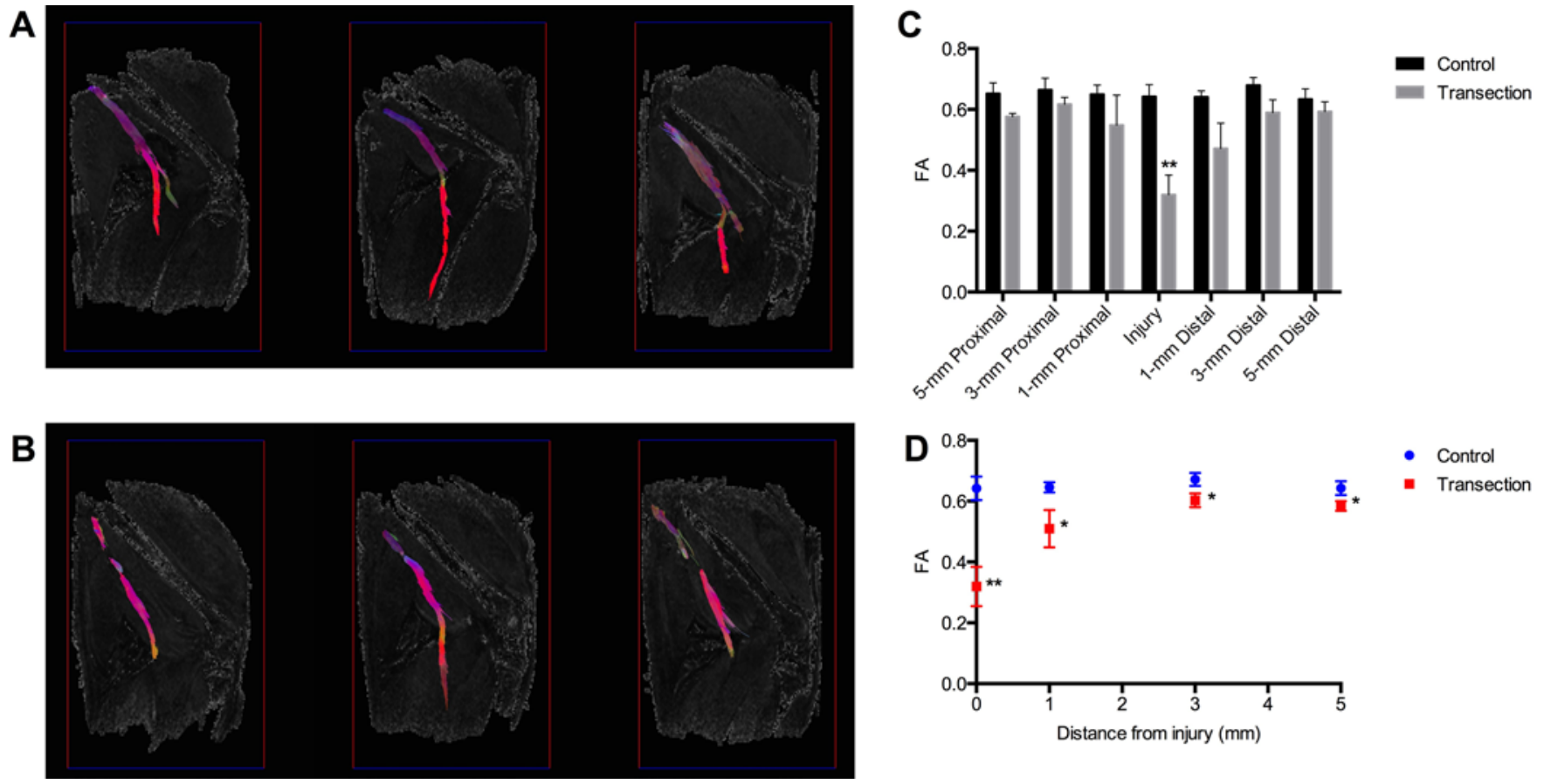

FIG. 6. FA remained sensitive to nerve transection in ex vivo hind limbs. A and B: Ex vivo diffusion tensor tractography of rat hind limbs with sham (A) and completely transected (B) sciatic nerves. C: FA measured at injury and each region proximal and distal to the injury (mean \pm SEM; $n=8$ ). D: FA approached sham values with increasing distance from the injury site but remained significantly lower at all points (mean $\pm S E M ; n=8$ ). Unpaired t-tests corrected for multiple comparisons using the Holm-Sidak method. ${ }^{*} p<0.05 ;{ }^{* *} p<0.01$.

isotropic diffusion, but should have minimal effect on relative tissue anisotropy. ${ }^{30}$ As observed in our hindlimb imaging, surrounding tissue and edema confounds the in vivo nerve DTI signal. Although FA remained an accurate marker of traumatic nerve injury, tractography capabilities were limited and required a different approach for tract seeding. Use of a multicompartment diffusion-MRI model such as diffusion basis spectrum imaging could isolate the confounding effects of edema and inflammation and improve nerve assessment. . $^{32,33}$

A second limitation of this study was the time required for high-resolution image acquisition-up to 12 hours for 1 set of 6 nerves. For translating this technology, it will be critical to find the resolution and FOV that minimize scan time while maintaining signal-to-noise ratio (SNR) and diagnostic accuracy. New high-resolution DTI methods such as outer-volume suppression and elliptically refocused zonally oblique multislice have been developed that leverage reduced-FOV imaging to significantly decrease data-set sizes and scan times up to 8-fold. ${ }^{13,34}$ Sensitivity encoding and other parallel imaging techniques can be used in combination with reduced-FOV techniques to further reduce data-set sizes, but their use is limited by SNR reduction.

\section{Conclusions}

The benefits of DTI for noninvasive assessment of axonal pathology have been well established in the central and peripheral nervous systems. This study provides confirmation that high-resolution DTI is capable of aiding acute diagnosis and grading of traumatic peripheral nerve injuries in a rat model. We have also provided an immediate baseline of DTI parameters following microsurgical coaptation of the sciatic nerve. Additional survival studies of traumatic peripheral nerve injury are needed to evaluate DTI as a tool for postoperative monitoring of nerve regeneration.

\section{Acknowledgments}

We thank Dr. Alexander Leemans for providing ExploreDTI software that was used for nerve tractography. This work was supported by Public Health Service award T32 GM07347 from the National Institute of General Medical Studies for the Vanderbilt Medical-Scientist Training Program, NIH/NIBIB grant EB001744, and NIH training grant T32 EB014841. This work was also supported by Department of Defense grant OR120216.

\section{References}

1. Aagaard BD, Lazar DA, Lankerovich L, Andrus K, Hayes $\mathrm{CE}$, Maravilla $\mathrm{K}$, et al: High-resolution magnetic resonance imaging is a noninvasive method of observing injury and recovery in the peripheral nervous system. Neurosurgery 53:199-204, 2003

2. Agosta F, Absinta M, Sormani MP, Ghezzi A, Bertolotto A, Montanari E, et al: In vivo assessment of cervical cord damage in MS patients: a longitudinal diffusion tensor MRI study. Brain 130:2211-2219, 2007

3. Bain JR, Mackinnon SE, Hunter DA: Functional evaluation of complete sciatic, peroneal, and posterior tibial nerve lesions in the rat. Plast Reconstr Surg 83:129-138, 1989

4. Beaulieu C, Does MD, Snyder RE, Allen PS: Changes in water diffusion due to Wallerian degeneration in peripheral nerve. Magn Reson Med 36:627-631, 1996

5. Behr B, Schnabel R, Mirastschijski U, Ibrahim B, Angenstein 
F, Schneider W: Magnetic resonance imaging monitoring of peripheral nerve regeneration following neurotmesis at 4.7 Tesla. Plast Reconstr Surg 123:1778-1788, 2009

6. Bendszus M, Stoll G: Technology insight: visualizing peripheral nerve injury using MRI. Nat Clin Pract Neurol 1:45-53, 2005

7. Bendszus M, Wessig C, Solymosi L, Reiners K, Koltzenburg M: MRI of peripheral nerve degeneration and regeneration: correlation with electrophysiology and histology. Exp Neurol 188:171-177, 2004

8. Bennett RE, Mac Donald CL, Brody DL: Diffusion tensor imaging detects axonal injury in a mouse model of repetitive closed-skull traumatic brain injury. Neurosci Lett 513:160165,2012

9. Campbell WW: Evaluation and management of peripheral nerve injury. Clin Neurophysiol 119:1951-1965, 2008

10. Cartwright MS, Chloros GD, Walker FO, Wiesler ER, Campbell WW: Diagnostic ultrasound for nerve transection. Muscle Nerve 35:796-799, 2007

11. Chhabra A, Andreisek G, Soldatos T, Wang KC, Flammang AJ, Belzberg AJ, et al: MR neurography: past, present, and future. AJR Am J Roentgenol 197:583-591, 2011

12. Filler AG, Howe FA, Hayes CE, Kliot M, Winn HR, Bell BA, et al: Magnetic resonance neurography. Lancet 341:659-661, 1993

13. Gaggl W, Jesmanowicz A, Prost RW: High-resolution reduced field of view diffusion tensor imaging using spatially selective RF pulses. Magn Reson Med 72:1668-1679, 2014

14. Gaudet AD, Popovich PG, Ramer MS: Wallerian degeneration: gaining perspective on inflammatory events after peripheral nerve injury. J Neuroinflammation 8:110, 2011

15. Grant GA, Britz GW, Goodkin R, Jarvik JG, Maravilla K, Kliot M: The utility of magnetic resonance imaging in evaluating peripheral nerve disorders. Muscle Nerve 25:314-331, 2002

16. Grant GA, Goodkin R, Kliot M: Evaluation and surgical management of peripheral nerve problems. Neurosurgery 44:825-840, 1999

17. Howe FA, Filler AG, Bell BA, Griffiths JR: Magnetic resonance neurography. Magn Reson Med 28:328-338, 1992

18. Krause TL, Bittner GD: Rapid morphological fusion of severed myelinated axons by polyethylene glycol. Proc Natl Acad Sci U S A 87:1471-1475, 1990

19. Kretschmer T, Heinen CW, Antoniadis G, Richter HP, König RW: Iatrogenic nerve injuries. Neurosurg Clin $\mathbf{N}$ Am 20:73-90, vii, 2009

20. Lee JY, Giusti G, Wang H, Friedrich PF, Bishop AT, Shin AY: Functional evaluation in the rat sciatic nerve defect model: a comparison of the sciatic functional index, ankle angles, and isometric tetanic force. Plast Reconstr Surg 132: $1173-1180,2013$

21. Leemans A, Jeurissen B, Sijbers J, Jones DK: ExploreDTI: a graphical toolbox for processing, analyzing, and visualizing diffusion MR data. Proc Intl Soc Mag Reson Med 17:3537, 2009 (Abstract)

22. Lehmann HC, Zhang J, Mori S, Sheikh KA: Diffusion tensor imaging to assess axonal regeneration in peripheral nerves. Exp Neurol 223:238-244, 2010

23. Li X, Chen J, Hong G, Sun C, Wu X, Peng MJ, et al: In vivo DTI longitudinal measurements of acute sciatic nerve traction injury and the association with pathological and functional changes. Eur J Radiol 82:e707-e714, 2013

24. Liang D, Bhatta S, Gerzanich V, Simard JM: Cytotoxic edema: mechanisms of pathological cell swelling. Neurosurg Focus 22(5):E2, 2007

25. Morisaki S, Kawai Y, Umeda M, Nishi M, Oda R, Fujiwara $\mathrm{H}$, et al: In vivo assessment of peripheral nerve regenera- tion by diffusion tensor imaging. J Magn Reson Imaging 33:535-542, 2011

26. Pham M, Wessig C, Brinkhoff J, Reiners K, Stoll G, Bendszus M: MR neurography of sciatic nerve injection injury. $\mathbf{J}$ Neurol 258:1120-1125, 2011

27. Riley DC, Bittner GD, Mikesh M, Cardwell NL, Pollins AC, Ghergherehchi CL, et al: Polyethylene glycol-fused allografts produce rapid behavioral recovery after ablation of sciatic nerve segments. J Neurosci Res 93:572-583, 2015

28. Simon NG, Kliot M: Diffusion weighted MRI and tractography for evaluating peripheral nerve degeneration and regeneration. Neural Regen Res 9:2122-2124, 2014

29. Suk JI, Walker FO, Cartwright MS: Ultrasonography of peripheral nerves. Curr Neurol Neurosci Rep 13:328, 2013

30. Sun SW, Neil JJ, Liang HF, He YY, Schmidt RE, Hsu CY, et al: Formalin fixation alters water diffusion coefficient magnitude but not anisotropy in infarcted brain. Magn Reson Med 53:1447-1451, 2005

31. Takagi T, Nakamura M, Yamada M, Hikishima K, Momoshima S, Fujiyoshi K, et al: Visualization of peripheral nerve degeneration and regeneration: monitoring with diffusion tensor tractography. Neuroimage 44:884-892, 2009

32. Wang X, Cusick MF, Wang Y, Sun P, Libbey JE, Trinkaus K, et al: Diffusion basis spectrum imaging detects and distinguishes coexisting subclinical inflammation, demyelination and axonal injury in experimental autoimmune encephalomyelitis mice. NMR Biomed 27:843-852, 2014

33. Wang Y, Wang Q, Haldar JP, Yeh F-C, Xie M, Sun P, et al: Quantification of increased cellularity during inflammatory demyelination. Brain 134:3590-3601, 2011

34. Wargo CJ, Gore JC: Localized high-resolution DTI of the human midbrain using single-shot EPI, parallel imaging, and outer-volume suppression at 7T. Magn Reson Imaging 31:810-819, 2013

35. Zhou Y, Kumaravel M, Patel VS, Sheikh KA, Narayana PA: Diffusion tensor imaging of forearm nerves in humans. J Magn Reson Imaging 36:920-927, 2012

\section{Disclosure}

The authors report no conflict of interest concerning the materials or methods used in this study or the findings specified in this paper.

\section{Author Contributions}

Conception and design: Riley, Boyer, Kelm, Sexton, Does, Thayer. Acquisition of data: Riley, Boyer, Kelm, Sexton, Pollins. Analysis and interpretation of data: Riley, Boyer, Sexton, Pollins, Dortch, Does, Thayer. Drafting the article: Boyer, Kelm, Thayer. Critically revising the article: Riley, Boyer, Kelm, Pollins, Dortch, Does, Thayer. Reviewed submitted version of manuscript: all authors. Approved the final version of the manuscript on behalf of all authors: Riley. Statistical analysis: Riley, Boyer, Kelm, Does. Administrative/technical/material support: Shack, Thayer. Study supervision: Boyer, Shack, Thayer.

\section{Supplemental Information}

\section{Previous Presentation}

Portions of this work were presented in abstract form at the American Society for Peripheral Nerve (ASPN) Annual Conference in January 2014.

\section{Correspondence}

D. Colton Riley, Department of Plastic Surgery, Vanderbilt University School of Medicine, 116121 st Ave. S, MCN S-221, Nashville, TN 37232. email: dcolton.riley@gmail.com. 